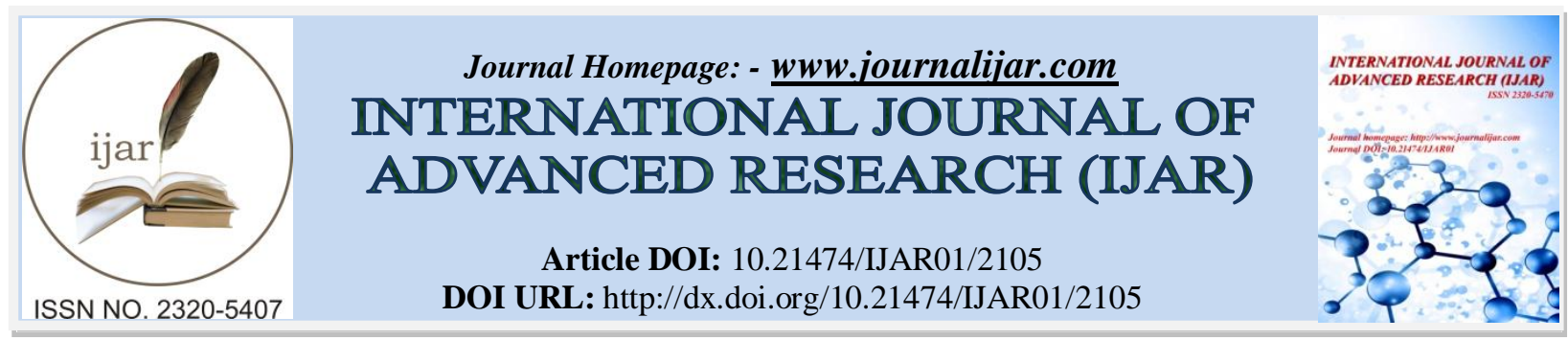

RESEARCH ARTICLE

\title{
ASSESSMENT OF IUI OUTCOME FOR INFERTILE COUPLES WITH MALE INFERTILITY USING SPERM MAX PURE VERSUS DENSITY GRADIENT TECHNIQUES.
}

\section{Prof. Dr. Muhammad Baqir M- R. Fakhrildin ${ }^{1}$, Lecturer Dr. Manal Al Obaidi, Dr. Zainb Hassan Hashim. And Prof. Dr. Mohammad Oda Selman.}

\section{Manuscript Info}

Manuscript History

Received: 24 September 2016

Final Accepted: 26 October 2016

Published: November 2016

Key words:-

engaged employee, employee in higher education, employee disengagement, corporate vs. educational culture

\section{Abstract}

Background:Different techniques are used for separation of motile and morphologically normal spermatozoa to be used for artificial insemination (AI) and other assisted reproductive techniques.Sperm preparation is important because some component of seminal fluid may become obstacle to fertilization when IUI or IVF are performed. However the choice of sperm selection technique depend on the quality of the semen in order to obtain the higher number of normal spermatozoa.

Objective: To assess the pregnancy rate for infertile couples undergoing IUI using Maxpure technique versus density gradient technique to prepare sperm from males with male infertility.

Subjects, Materials and Methods: One hundred twenty seven (127) infertile couples participated in this study during their attendance to the infertility clinics at the High Institute for Infertility Diagnosis and Assisted Reproductive Technologies, Al-Nahrain University. The period of study was from September 2015 to June 2016. The mean age for all males included in this study was $35.33 \pm 0.731$ and the mean age for all females included in the study was $30.67 \pm 0.711$ years. The mean duration of infertility was $6.18 \pm 0.403$ ranged from 1- 21 years.

Semen analysis was done on the collected semen samples according to WHO (2010). From each semen sample two $\mathrm{mL}$ was taken. One $\mathrm{mL}$ was prepared using density gradient centrifugation technique, and the other one $\mathrm{mL}$ prepared using sperm Maxpure technique. Then sperm parameters were assessed for these two techniques, and the part with the best result had been used for intrauterine insemination and finally the pregnancy results were statistically analyzed.

Results: The reduction in sperm concentration was significantly $(\mathrm{P}<0.001)$ far more using Density gradients technique, mean progressive motile sperm $(\%)$ was significantly $(\mathrm{P}<0.001)$ more raised using sperm Max pure technique. However non-significant change $(\mathrm{P}=0.430)$ was observed regarding mean non progressive motile sperm (\%). Mean immotile sperm (\%) was far less using sperm Max pure technique $(\mathrm{P}<0.001)$.In contrast mean normal sperm morphology $(\%)$ was significantly higher using sperm Max pure technique $(\mathrm{P}<0.001)$. Finally, the round cell count/HPF was significantly $(\mathrm{P}<0.001)$ less using sperm Max pure technique. 
The percentage of pregnancy was $9.4 \%$, the number of pregnant woman was 11 out of 117. Nine cases of pregnancy resulted from using sperm Maxpure technique for sperm activation to be used for IUI, while using density gradient centrifugation technique for sperm activation resulted in two cases of pregnancy.

Conclusions:The sperm parameters outcome when using sperm Maxpure technique were superior to the outcome of density gradient centrifugation technique when using low quality semen samples. The rate of preparation failure was less when using sperm Maxpure technique as compared to density gradient centrifugation technique. The percentage of pregnancy was greater when using sperm Maxpure technique as compared to Density gradient centrifugation technique.

Copy Right, IJAR, 2016,. All rights reserved.

\section{Introduction:-}

A spermatozoon is a male germ cell, characterized by a small amount of cytoplasm and the most densely packed DNA known in eukaryotes.Spermatozoa are generated in the walls of loops of convoluted seminiferous tubules, formed from the primitive germ cells (by a process called spermatogenesis) ${ }^{(1)}$.

Semen analysis is an imperfect tool but still the cornerstone of the investigation of male infertility ${ }^{(2)}$. Routine semen analysis provides beneficial information concerning sperm production, sperm motility and viability, also patency of the male genital tract, secretions of the accessory organs, as well as ejaculation and emission ${ }^{(3)}$.

Artificial Insemination (AI) is the first option management for infertile couples with cervical factor sub-fertility, mild-moderate male sub-fertility and unexplained infertility ${ }^{(4,5)}$. The AI, as other assisted reproductive techniques, require a selection of the ejaculated sperm before the performance of the treatment. In fact, many components of the seminal fluid may become an obstacle to the fertilization when the In vitro fertilization (IVF) or the intrauterine insemination ( IUI) are performed ${ }^{(6)}$. Some different techniques are performed to prepare the spermatozoa for the AI, but the choice greatly depend on the quality of the semen, that mean it depend on the concentration, motility and morphology, in order to get the higher number of good spermatozoa, even from the bad quality semen ${ }^{(7)}$.

Intrauterine insemination(IUI) : is a type of artificial insemination, is a method for treating infertility. Sperm that have been washed and concentrated are introduced directly in the uterus around the time that the ovary releases one or more eggs to be fertilized. IUI may be done with or without ovulation induction (natural cycle) ${ }^{(8)}$.

So, the selection of the spermatozoa from the other components is performed by methods of sperm preparation techniques like the swim up technique or the gradient density centrifugation technique, glass wool filteration technique and sperm Maxpure technique ${ }^{(9)}$. Thereforethe objective of this study wasto assess the pregnancy rate for infertile couples undergoing IUI using Maxpure technique versus density gradient technique to prepare sperm from males with male infertility.

\section{Subjects, Materials and Methods:- \\ Subjects:-}

One hundred twenty seven (127) infertile couples participated in this study during their attendance to the Infertility Clinics at the High Institute for Infertility Diagnosis and Assisted Reproductive Technologies, Al-Nahrain University. The period of study was from September 2015 to June 2016. History including age, type of infertility, and duration of infertility were reported. The mean age for all males included in this study was $35.33 \pm 0.731$ years with a range from 20 to 55 years and the mean age for all females included in the study was $30.67 \pm 0.711$ years with a range from 17 - 47 years.

\section{Semen analysis:-}

Sample collection: The sample was collected in a private room near the laboratory, after a minimum of 2 days and a maximum of 5 days of sexual abstinence. The container was labeled with the following information, couples name, age, wife's name,abstinence period and time of sample collection. The sample obtained by masturbation and ejaculated into a clean, dry, wide-mouthed disposable Petri-dish. 
The specimen containers were placed in an incubator at $37{ }^{\circ} \mathrm{C}$ for $30-60$ minutes to allow for liquefaction. The liquefied semen was mixed for few second and examined by macroscopic and microscopic examination. The standard form of WHO (2010) is used to record the result of semen analysis ${ }^{(10)}$.

\section{Techniques of in vitro sperm activation:- \\ Density gradient centrifugation (DGC) Technique:-}

This technique done by adding one $\mathrm{ml}$ of $80 \%$ of Sil-Select Plus gradient as a first layer solution in a test tube followed by one $\mathrm{ml}$ of $40 \%$ of Sil-Select Plus gradient as a second layer solution then liquefied semen sample was added on the second layer. This test tube was carefully put in centrifuge at $2500 \mathrm{rpm}$ for 13 minutes. Then, the supernatant was discarded and one $\mathrm{ml}$ of Ferticult Flushing medium was added to the pellet and put in an air incubator for 30 minutes at $37^{\circ} \mathrm{C}$. A drop of $10 \mu \mathrm{l}$ was aspirated and put on a slide with cover slip and examined under the microscope at $400 \mathrm{X}$ magnification power to assess the sperm parameters.

\section{Sperm Maxpure Technique:-}

This technique done by adding one $\mathrm{ml}$ of $80 \%$ of Sil-Select Plus gradient as a first layer solution in a test tube followed by one $\mathrm{ml}$ of $40 \%$ of Sil-Select Plus gradient as a second layer solution, and then liquefied semen sample was added on the second layer. This test tube was carefully put in centrifuge at $2500 \mathrm{rpm}$ for 13 minutes. Then the supernatant was discarded and one $\mathrm{ml}$ of Ferticult Flushing medium was added to the pellet. Then shaking the sample and waiting for 8-10 minutes in an incubator, after that the semen suspension was placed gently over the wet glass wool syringe and allowed to filter by gravity. A drop of $10 \mu \mathrm{l}$ was aspiratedfrom filterate, put on a slide with cover slip and examined under the microscope at 400X magnification power to assess the sperm parameters. Finally, this technique was invented by Prof. Dr. Muhammad-Baqir M-R. Fakhrildin in $2013^{(11)}$.

\section{Intrauterine Insemination (IUI):-}

Ovulation Induction Program for IUI:-

- Induction of ovulation by clomiphene citrate (Clomid tablet $50 \mathrm{mg}$, Aventis France) twice daily for 5 days from cycle day 2-6.

- induction of ovulation by r-hFSH (Gonal- $\mathrm{F}^{\circledR}$, Serono-Italy) as subcutaneous injection from CD3 and the dose was adapted depending on the ovarian response to the treatment as detected by a serial US examination ${ }^{(12)}$.

- clomiphene citrate $(50 \mathrm{mg})$ twice daily was given from CD2-6 followed by $\mathrm{rFSH}\left(\right.$ Gonal- $\mathrm{F}^{\circledR}$ ) for two or more injection which was adjusted depending on ovarian response ${ }^{(13)}$ till follicular maturity $(17-23 \mathrm{~mm})$ was followed by hCG administration, Ovitrelle ${ }^{\circledR}$ injection $250 \mu \mathrm{g}$ one or two ampoules if more than one follicles, then insemination was performed after 30-36 hours.

- clomiphene citrate $(50 \mathrm{mg}$ ) twice daily was given from CD2-6 followed by human menopausal gonadotrpins (Menogon) injections intramuscularly from cycle day 3 through the follicular phase either daily or every other day $^{(14)}$.

- Follicular maturity was monitored by a serial ultrasound (vaginal US to optimize image quality), as well as, evaluating follicle number, size, endometrial thickness and patterns.

The choice of stimulation protocol was individualized according to patient age, diagnosis, reproductive history, level of basal serum hormones, ovarian response and coexisting medical conditions.

\section{Procedure of Intrauterine insemination (IUI):-}

The female partner was prepared for IUI, which was performed in a gynecological room with a clean sterile technique,by a special intrauterine catheter attached with $2 \mathrm{~mL}$ syringe containing the insemination volume of 0.5 $\mathrm{ml}$ of activated sperm sample. A Cusco's speculum was placed in the vagina to visualize the cervix. The patient remained in supine position for 30 minutes after the procedure ${ }^{(15)}$. In a stimulated cycle if HCG is administered when the average diameter of the dominant follicle is $17-20 \mathrm{~mm}$, rupture of the follicle may be expected within 3036 hours. Luteal phase support started from next day for two weeks by giving the patient duphastone $₫ 10 \mathrm{mg}$ twice daily $^{(16)}$. Estimation of Beta human chorionic gonadotropin $(\beta-h C G)$ was carried out on day 16 after IUI for pregnancy confirmation.

\section{Experimental Design:-}

The present study was performed on one hundred twenty seven infertile couples. Semen samples were collected and semen analysis was done according to WHO (2010). Each semen sample was divided into two aliquot. One division prepared by density gradient centrifugation technique, the other one prepared by Max pure technique (combination of density gradient centrifugation and glass wool filtration techniques). Then sperm parameters were assessed and 
compared. The portion of the semen sample that gave best results for sperm parameter was used for intra uterine insemination (IUI) of female partner.

\section{Statistical analysis:-}

The data were statistically analyzed using Statistical Package for Social Sciences (SPSS) version 22 software.

Numerical variables were expressed as mean \pm standard error of mean where as nominal variables were expressed as numbers and percentages. Paired $t$-test and chi square $\times 2$ test were applied to compare among different groups of in vitro sperm activation techniques. The difference between values of means was considered statistically significant when $\mathrm{P}<0.05$.

\section{Results:-}

One hundred and twenty seven (127) couples were included in this study. All males have undergone the process of sperm preparation using two techniques, Density gradient and sperm maxpure.

In six cases, the sperm activation was failed (four cases by using DGC, one case with using sperm maxpure and the last case failed after using both techniques) . In the rest cases (121), the female partner were subjected to IUI using the best husbands sample resulted from the two types of sperm activation mentioned above.

Four couples of IUI cases did not give the IUI results (no answer). Finally, one hundred seventeen cases only were included in analysis of IUI results.

There was significant $(\mathbf{P}<0.001)$ reduction of sperm concentration after using sperm Maxpure technique, significant $(\mathbf{P}=0.001)$ increase in progressive sperm motility (\%), significant reduction of non progressive sperm motility (\%) $(\mathbf{P}=0.031)$, immotile sperm (\%) $(\mathbf{P}<0.001)$, round cell/HPF $(\mathbf{P}=0.002)$, sperm agglutination (\%) $(\mathbf{P}=0.035)$, also there was significant $(\mathbf{P}=0.001)$ increase in normal sperm morphology $(\%)$

Sperm activation using Density gradient technique resulted in significant $\left(\mathbf{P}^{\prime}<0.001\right)$ reduction in sperm concentration, significant $\left(\mathbf{P}^{\prime}=0.003\right)$ increase in progressive sperm motility $(\%)$, significant $\left(\mathbf{P}^{\prime}=0.017\right)$ reduction of non progressive sperm motility $(\%)$, immotile sperm $(\%)\left(\mathbf{P}^{\prime}=0.019\right)$, round cell count $\left(\mathbf{P}^{\prime}=0.001\right)$, sperm agglutination (\%) $(\mathbf{P}=0.035)$, significant $\left(\mathbf{P}^{\prime}=0.004\right)$ increase in normal sperm morphology $(\%)$, as shown in table (1).

Table 1:- Sperm parameters in normozoospermic group after activation by Maxpure and Density gradient centrifugation techniques.

\begin{tabular}{|c|c|c|c|c|c|c|}
\hline \multirow{2}{*}{\multicolumn{2}{|c|}{ Sperm parameters }} & \multirow{2}{*}{$\begin{array}{l}\text { Pre activation } \\
\text { Mean } \pm \text { SEM }\end{array}$} & \multicolumn{4}{|c|}{ Post Activation Mean \pm SEM } \\
\hline & & & Density Gradient & $\begin{array}{l}\text { P- } \\
\text { value }\end{array}$ & Maxpure & $\begin{array}{l}P^{\prime} \text { - } \\
\text { value }\end{array}$ \\
\hline \multicolumn{2}{|l|}{ Concentration } & $16.75 \pm 0.56$ & $8.75 \pm 1.08$ & $<0.001$ & $9.63 \pm 0.53$ & $<0.001$ \\
\hline \multirow[t]{4}{*}{ Sperm Grade Activity (\%) } & Progressive & $47.13 \pm 3.20$ & $64.63 \pm 1.20$ & 0.001 & $59.50 \pm 1.04$ & 0.003 \\
\hline & Non Progressive & $36.75 \pm 3.03$ & $28.75 \pm 1.32$ & 0.031 & $27.63 \pm 1.64$ & 0.017 \\
\hline & Total & $83.88 \pm 0.61$ & $93.38 \pm 0.37$ & $<0.001$ & $87.13 \pm 0.86$ & 0.019 \\
\hline & Immotile & $16.13 \pm 0.61$ & $6.63 \pm 0.37$ & $<0.001$ & $12.88 \pm 0.86$ & 0.019 \\
\hline \multicolumn{2}{|c|}{ Normal Sperm Morphology (\%) } & $43.75 \pm 2.24$ & $53.63 \pm 2.78$ & 0.001 & $56.88 \pm 2.66$ & 0.004 \\
\hline \multicolumn{2}{|c|}{ Round cell/HPF } & $3.00 \pm 0.60$ & $0.13 \pm 0.12$ & 0.002 & $0.00 \pm 0.00$ & 0.001 \\
\hline \multicolumn{2}{|l|}{ Sperm Agglutination (\%) } & $4.50 \pm 1.72$ & $0.00 \pm 0.00$ & 0.035 & $0.00 \pm 0.00$ & 0.035 \\
\hline
\end{tabular}

With the exception of sperm agglutination (\%) that showed great decrease but statistically non significant, there was significant $\left(\mathbf{P}^{\prime}<0.001\right)$ reduction in sperm concentration, significant $\left(\mathbf{P}^{\prime}<0.001\right)$ increase in progressive sperm motility $(\%)$, significant $\left(\mathbf{P}^{\prime}=0.013\right)$ decrease in non progressive sperm motility $(\%)$, significant $\left(\mathbf{P}^{\prime}<0.001\right)$ decrease in immotile sperm $(\%)$ and round cell/HPF $\left(\mathbf{P}^{\prime}<0.001\right)$ and there was significant $\left(\mathbf{P}^{\prime}<0.001\right)$ increase in normal sperm morphology (\%) as shown in table(2). Similarly significant changes were reported in all sperm parameters after density gradient activation in oligozoospermic group, excluding sperm agglutination (\%) which showed nonsignificant $\left(\mathbf{P}^{\prime}=0.072\right)$ reduction, as shown in table $(2)$. 
Table 2:- Sperm parameters in oligozoospermia group after activation by Maxpure and Density gradient centrifugation techniques.

\begin{tabular}{|c|c|c|c|c|c|c|}
\hline \multirow{2}{*}{\multicolumn{2}{|c|}{ Sperm parameters }} & \multirow{2}{*}{$\begin{array}{l}\text { Pre- activation } \\
\text { Mean } \pm \text { SEM }\end{array}$} & \multicolumn{4}{|c|}{ Post-activation } \\
\hline & & & $\begin{array}{l}\text { (Density } \\
\text { Gradient } \\
\text { Mean } \\
\text { SEM }\end{array}$ & $\begin{array}{l}P \text { P- } \\
\text { value }\end{array}$ & $\begin{array}{l}\text { Maxpure } \\
\text { Mean } \pm \text { SEM }\end{array}$ & $\begin{array}{l}P^{\prime}- \\
\text { value }\end{array}$ \\
\hline \multicolumn{2}{|l|}{ Concentration } & $10.81 \pm 0.61$ & $3.86 \pm 0.42$ & $<0.001$ & $5.43 \pm 0.49$ & $<0.001$ \\
\hline \multirow[t]{4}{*}{ Sperm Grade Activity (\%) } & Progressive & $58.76 \pm 2.98$ & $46.24 \pm 1.66$ & $<0.001$ & $63.76 \pm 2.51$ & $<0.001$ \\
\hline & Non Progressive & $25.24 \pm 1.44$ & $32.14 \pm 1.20$ & 0.001 & $26.67 \pm 1.55$ & 0.013 \\
\hline & Total & $84.00 \pm 2.75$ & $78.38 \pm 1.96$ & 0.018 & $90.43 \pm 1.24$ & $<0.001$ \\
\hline & Immotile & $16.00 \pm 2.75$ & $21.14 \pm 1.92$ & 0.020 & $9.57 \pm 1.24$ & $<0.001$ \\
\hline \multicolumn{2}{|c|}{ Normal Sperm Morphology (\%) } & $32.57 \pm 1.65$ & $38.86 \pm 1.84$ & $<0.001$ & $41.24 \pm 1.96$ & $<0.001$ \\
\hline \multicolumn{2}{|c|}{ Round cell/HPF } & $4.81 \pm 0.73$ & $0.10 \pm 0.07$ & $<0.001$ & $0.00 \pm 0.00$ & $<0.001$ \\
\hline \multicolumn{2}{|l|}{ Sperm Agglutination (\%) } & $0.38 \pm 0.20$ & $0.00 \pm 0.00$ & 0.072 & $0.00 \pm 0.00$ & 0.072 \\
\hline
\end{tabular}

The overall rate of pregnancy after IUI obtained in this study was $9.4 \%$.Nine (9) cases of pregnancy resulted from using sperm Maxpure technique for sperm activation to be used for IUI , while using density gradient centrifugation technique for sperm activation resulted in two cases of pregnancy. As shown in the figure (1).

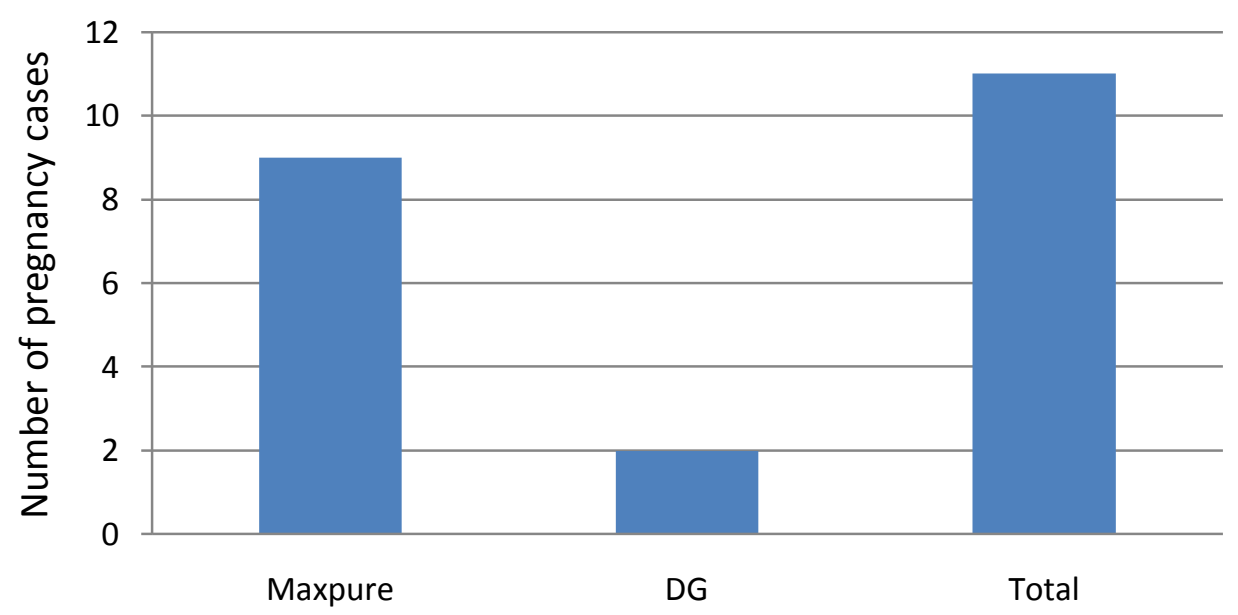

Figure 1:- Number of pregnancy cases resulted by Maxpure and Density Gradient

\section{Discussion:-}

With ART, the semen samples must be dealt with before they can be used for insemination. Sperm preparation techniques are necessary to remove non-motile spermatozoa, leukocyte, prostaglandins, antigenic proteins, infectious agents, and immature germ cells. These processing techniques provides better sperm quality regarding concentration and motility, which may lead to higher success rates after IUI ${ }^{(17)}$.

During sperm preparation technique not only progressively motile spermatozoa are selected, but sperm cells also undergo physiological changes called capacitation, which are fundamental prerequisites for the sperm's functional competence with regard to acrosome reaction ${ }^{(17,18)}$.

In this study most semen sample that had been involved represented different types of male infertility factors, so the choice of DGC and sperm Maxpure technique were suitable for these samples because these two methods were the best for activation of bad quality semen samples, that may had reduction in sperm concentration and sperm motility , increase percentage of abnormal sperms morphology or combination of these abnormalities ${ }^{(11,19,20)}$. Therefore The need for effective sperm preparation method has increased with increasing use of ART . For better sperm activation result, the choice of activation method depend on the quality of ejaculate ${ }^{(7)}$. 
This study clarified a significant reduction $(\mathrm{P}<0.001)$ for the sperm concentration after sperm preparation when using the two techniques for all types of ejaculate as compared to pre-activation this agreed with Kouty (2007) ${ }^{(21)}$. The reduction in sperm concentration by using density gradient centrifugation technique was due to the different densities of the media that isolate normal sperm from sperm with low motility, immotile sperm or sperm with abnormal morphology. While in sperm Max pure technique in addition to previous step, the glass wool fibers held back the abnormal spermatozoa by adhesion. Also, the sperm Maxpure Technique gave higher concentration than DGC technique, because of pellet formation that may impedes movement of some sperms, while in Maxpure technique the mixing of pellet resulted in maximum percentage of moving sperm ${ }^{(22)}$.

The present work showed a significant increase $(\mathrm{P}<0.001)$ for progressive sperm motility after sperm preparation when using Maxpure technique as compared to DGC and also, clarified a significant increase $(\mathrm{P}<0.001)$ for the same parameter when using DGC for sperm activation as compared to pre-activation. The aim of doing sperm activation is to improve sperm motility and fertilizing capacity, and this improvement resulted from removing seminal plasma with its content of dead sperm, leukocyte , immature germ cells, and others that might produce reactive oxygen species, also eliminate sperm agglutination that resulted from the presence of anti-sperm antibodies that can negatively affect the fertilization process ${ }^{(24,25)}$.

The current study clarified significant improvement $(\mathrm{P}<0.001)$ for normal sperm morphology when using DGC as compared to pre-activation. Also, showed a significant improvement $(\mathrm{P}<0.001)$ for normal sperm morphology when using sperm Maxpure technique as compared to DGC technique for all types of ejaculate were studied. Sperm morphology is important indicator for the testicular health and it's function because the sperm morphology is determined by process of spermatogenesis ${ }^{(26)}$, Belaisch-Allhart et al., suggested that no IUI should be carried out with a teratozoospermia rate over $80 \%{ }^{(27)}$, additionally by sperm activation techniques sperm morphology percentage improved and this subsequently improve the sperm motility which in turn affect the fertilization process positively. and this agreed with Burr Ret $\mathrm{al}^{(28,29)}$.

Significant reduction $(\mathrm{P}<0.001)$ in the agglutination of sperm were obtained follow the activation by sperm Maxpure and DGC techniques as compared to pre-activation for all types of ejaculate in the study. Sperm agglutination refer mostly to presence of anti-sperm antibodies which cause sticking of sperm to each other in a variable degree and this cause great limitation of sperm motility which affect the fertilization process greatly ${ }^{(30)}$.

The overall rate of pregnancy after IUI obtained in this study was $9.4 \%$, This agreed with many studies like Tredway DR, deMouzon, et al ${ }^{(27,28)}$.Nine (9) cases of pregnancyresulted from using sperm Maxpure technique for sperm activation to be used for IUI, while using density gradient centrifugation technique for sperm activation resulted in two cases of pregnancy, total number of pregnant women was 11 out of 117 female that subjected to IUI.

From The result of present study it was concluded that:

sperm Max pure technique was better than density gradient centrifugation technique by achieving significant enhancement of the progressive sperm motility and normal sperm morphology for all types of ejaculate were studied.

The percentage of pregnancy was greater when using sperm Maxpure technique as compared to density gradient technique.

Chairman of clinical physiology Dept., faculty of medicine, Jabir Ibn Hayyan , medical university, Al-Najaf AlAshraf.

Head of reproductive physiology Dept., High institute of infertility diagnosis and assisted reproductive technology, Al- Nahrain university, Baghdad

\section{References:-}

1. Geijsen N, Daley GQ, Male germ cells, Methods Enzymol, 2006;418: 307-14.

2. Boomsma, C.M.; Heineman, M.J., Cohlen, B.J. \& Farquhar, C., Semen preparation techniques for intrauterine insemination. Cochrane Database of Systematic Reviews 2004; (3):CD004507

3. Rune Eliasson.Semen analysis with regard to sperm number, sperm morphology and functional aspects Asian Journal of Andrology (2010) 12: 26-32.

4. MiladManafi, "Artificial Insemination in Farm Animals", Veterinary Medicine and Science, June 21, 2011 , Chapter 7. 
5. Bensdorp AJ, Cohlen BJ, Heineman MJ, Vandekerckhove P., Intra- Uterine Insemination for male subfertility. Cochrane Database Syst Rev 2007; Art No.: CD000360.

6. Tohoku J. "Methods of semen preparation for intrauterine insemination and subsequent pregnancy rates", Exp Med. 1992 Dec;168(4):583-90.

7. Ombelet $\mathrm{W}^{1}$, Deblaere K, Bosmans E, Cox A, Jacobs P, Janssen M, Nijs M.Zavos PM,Centola GM. Semen quality and intrauterine insemination, Reprod Biomed Online. 2003 Oct-Nov;7(4):485-92.

8. Aitken, R.J. \& Clarkson, J.S., Significance of Reactive Oxygen Species and Antioxidants in Defining the Efficacy of Sperm Preparation Techniques. J of Androl, 1988, Vol.9, No.6, pp.367-76.

9. Mohan S Kamath, PriyaBhave, TK Aleyamma, Raju Nair, ${ }^{1}$ A Chandy, Ann M Mangalaraj, K Muthukumar, and Korula George, Predictive factors for pregnancy after intrauterine insemination: A prospective study of factors affecting outcome, J Hum Reprod Sci. 2010 Sep-Dec; 3(3): 129-134.

10. World Health Organization. WHO Laboratory Manual for the

11. Examination and Processing of Human Semen, 5thed. Geneva:WHO Press 2010.

12. Muhammad Baqir M. R. Fakhrildin, Ula M. R. Al-Kawaz and EzdeharNassif Ali, A New Sperm Preparation Technique by combination of Density Gradient Centrifugation and Glass Wool Filtration Techniques Versus Each One Alone for Infertile Males. Master Thesis, Al-Nahrain University. 2015.

13. Peter Y. Liu, Leo Turner, Debra Rushford, Jillian McDonald, H.W.Gordon Baker, Ann J. Conway and David J. Handelsman 1, Efficacy and safety of recombinant human follicle stimulating hormone (Gonal-F) with urinary human chorionic gonadotrophin for induction of spermatogenesis and fertility in gonadotrophin-deficient men, Oxford JournalsMedicine \& HealthHuman Reproduction Volume 14,Issue 6 Pp. 1540-1545.

14. Monika Weigert, Ursula Krischker, Michaela Pöhl, GundaPoschalko, ChristophKindermann, WilfriedFeichtinger, Comparison of stimulation with clomiphene citrate in combination with recombinant follicle-stimulating hormone and recombinant luteinizing hormone to stimulation with a gonadotropin-releasing hormone agonist protocol: a prospective, randomized study,Fertility and SterilityVolume 78, Issue 1, July 2002, Pages 34-39.

15. Abdelazim IA ${ }^{1}$, Makhlouf HH, Sequential clomiphene citrate/hMG versus hMG for ovulation induction in clomiphene citrate-resistant women, Arch Gynecol Obstet. 2013;287(3):591-7.

16. W. Ombelet, P. Puttemans and E. Bosmans, Intrauterine insemination: a first-step procedure in the algorithm of male subfertility treatment, Oxford Journals, Medicine \& Health, Human Reproduction, Volume 10,Issue suppl 1 ,Pp. 90-102

17. Caroline E. Overton, Peter C. Lindsay, BalroopJohal, Susan A. Collins, Nicholas C. Siddle, Robert W. Shaw, A randomized, double-blind, placebo-controlled study of luteal phase dydrogesterone (Duphaston) in women with minimal to mild endometriosist, Fertility and Sterility ,Volume 62, Issue 4, 1994, Pages 701-707.

18. Bedford JM., Significance of the need for sperm capacitation before fertilization in eutherian mammals; BiolReprod. 1983;28:108-120. [PubMed]

19. David H. Barlow, M.D.Yanagimachi R. Knobil E, Neill JD, Ewing LL, Markert CL, Greenwald GS, Pfaff DW, The Physiology of Reproduction. Vol. 1. Raven Press, New York; 1988. pp. 135-185.

20. J. Rhemrev, R. S. Yejendran, J. P. Vermeiden, L. J. Zaneveld, 1989 Human sperm selection by glass wool filtration and two-layer, discontinuous Percoll gradient centrifugation. FertilSteril, 514 (April 1989), 685690

21. K. Sterzik, M. De Santo, S. Uhlich, F. Gagsteiger, E. Strehler, 1998 Glass wool filtration leads to a higher percentage of spermatozoa with intact acrosomes: an ultrastructural analysis. Hum Reprod, 139 (September 1998), 25062511

22. Kouty BK. An evaluation of Hypo-osmotic Swelling Test Regarding : seminal fluid analysis , sperm preparation techniques and intra uterine insemination in infertile patients, Master of Science thesis in Applied Embryology, Institute of Embryo Research and infertility Treatment ,University of Al- Nahrain ,2007.

23. PoenickeK, Grunewald S, GlanderH, Paasch U., Sperm Selection in Assisted Reproductive Techniques. Rao KA, Agarwal A, Srinivas MS, Andrology Laboratory Manual. 1st edn. India: Jaypee Brothers Pvt Ltd 2010.pp.173-87.

24. J. R. Aitken, E. Gordon, D. Harkiss, J. P. Twigg, P. Milne, Z. Jennings, D. S. Irvine, 1998 Relative Impact of Oxidative Stress on the Functional Competence and Genomic Integrity of Human Spermatozoa. Biol of Reprod, 59 (1998), 10371046.

25. Cross, N.L. (1993) Multiple effects of seminal plasma on the AR of human sperm. Mol. Reprod. Dev., 35, 316323.

26. Agarwal A et al. Clinical relevance of oxidative stress in male factor infertility: an update. Am $\mathbf{J}$ ReprodImmunol. 2008;59(1):2-11. 
27. Badawy A, Elnashar A, Eltotongy M., Effect of sperm morphology and number on success of intrauterine insemination, FertilSteril. 2009;91(3):777-81.

28. Belaisch-Allart J, Mayenga JM, Plachot M., Intrauterine insemination. ContraceptFertil Sex 1999; 27: 616-621.

29. Merviel P, Heraud MH, Grenier M, Lourdel E, Sanguinet P and Coping H., " Predcitive factors for pregnancy after intrauterine insemination (IUI): An analysis of 1038 cycles and a review of the literature." FertilSteril 2010; 93: 79-88.

30. Burr R, Siegberg R, Mathews C,Flaherty S. The influence of spermatozoa morphology and the number of motile spermatozoa inseminated on the outcome of intrauterine insemination combined with mild ovarian stimulation. FertilSteril 1996; 65:127-132.

31. SetuMazumdar, M.D., Adam S Levine, M.D.Antisperm antibodies: etiology, pathogenesis, diagnosis, and treatment .Fertility and Sterility, Volume 70, Issue 5, November 1998, Pages 799-810.

32. Tredway DR, Chan P, Henig I, Gullett A, Cheatwood M., Effectiveness of stimulated menstrual cycles and Percoll sperm preparation in intrauterine insemination, J Reprod Med. 1990 Feb; 35(2):103-8.)

33. de Mouzon J, Goossens V, Bhattacharya S, Castilla JA, A.P. Ferraretti AP, Korsak V, Kupka M, Nygren KG, Nyboe Andersen A, The European IVF-monitoring (EIM) Consortium, for the European Society of Human Reproduction and Embryology (ESHRE). Assisted reproductive technology in Europe, 2006: results generated from European registers by ESHRE. Hum Reprod 2010; 25:1851-1862. 